alone and in cooperation with Meis1a in a murine model. Proc. Natl. Acad. Sci. U. S. A. 103:16924-16929.

18. Partanen, J., Schwartz, L., and Rossant, J. 1998. Opposite phenotypes of hypomorphic and Y766 phosphorylation site mutations reveal a function for Fgfr1 in anteroposterior patterning of mouse embryos. Genes Dev. 12:2332-2344.

19. Roche, J., et al. 2004. Hox expression in AML identifies a distinct subset of patients with intermediate cytogenetics. Leukemia. 18:1059-1063.

20. Muller-Tidow, C., et al. 2004. High-throughput analysis of genome-wide receptor tyrosine kinase expression in human cancers identifies potential novel drug targets. Clin. Cancer Res. 10:1241-1249.

21. Veraksa, A., Del Campo, M., and McGinnis, W. 2000. Developmental patterning genes and their conserved functions: from model organisms to humans. Mol. Genet. Metab. 60:85-100.

\title{
Hypoglycemia, functional brain failure, and brain death
}

\author{
Philip E. Cryer \\ Division of Endocrinology, Metabolism and Lipid Research, Washington University School of Medicine, St. Louis, Missouri, USA.
}

\begin{abstract}
Hypoglycemia commonly causes brain fuel deprivation, resulting in functional brain failure, which can be corrected by raising plasma glucose concentrations. Rarely, profound hypoglycemia causes brain death that is not the result of fuel deprivation per se. In this issue of the JCI, Suh and colleagues use cell culture and in vivo rodent studies of glucose deprivation and marked hypoglycemia and provide evidence that hypoglycemic brain neuronal death is in fact increased by neuronal NADPH oxidase activation during glucose reperfusion (see the related article beginning on page 910). This finding suggests that, at least in the setting of profound hypoglycemia, therapeutic hyperglycemia should be avoided.
\end{abstract}

Hypoglycemia, including iatrogenic hypoglycemia in people with diabetes, causes brain fuel deprivation that initially triggers a series of physiological and behavioral defenses but if unchecked results in functional brain failure that is typically corrected after the plasma glucose concentration is raised. Rarely, profound, and at least in primates prolonged, hypoglycemia causes brain death.

Given the survival value of maintaining physiological plasma glucose concentrations, it is not surprising that mechanisms that normally very effectively prevent or rapidly correct symptomatic hypoglycemia have evolved (1). As a result, hypoglycemia is a distinctly uncommon clinical event except in people who use drugs that lower the plasma glucose concentration (2). Although there are other drugs, and several relatively uncommon conditions, that cause hypoglycemia (2), in the vast majority of instances the offending drug is an insu-

Nonstandard abbreviations used: T1DM, type 1 diabetes mellitus.

Conflict of interest: The author has served on advisory boards of Novo Nordisk Inc., Takeda Pharmaceuticals North America Inc., MannKind Corp., and Merck and Co. and as a consultant to TolerRx Inc., Amgen Inc., and Marcadia Biotech in recent years.

Citation for this article: J. Clin. Invest. 117:868-870 (2007). doi:10.1172/JCI31669. lin secretagogue or insulin used to treat diabetes mellitus $(2,3)$. As a result of the interplay of relative or absolute therapeutic insulin excess and compromised physiological and behavioral defenses against falling plasma glucose concentrations, hypoglycemia is the limiting factor in the glycemic management of diabetes (3). It causes recurrent morbidity in most people with type 1 diabetes mellitus (T1DM) and in many with advanced T2DM and is sometimes fatal. Furthermore, hypoglycemia, as well as prior exercise and sleep, further compromise glycemic defenses by causing hypoglycemia-associated autonomic failure and thus a vicious cycle of recurrent hypoglycemia. Finally, the barrier of hypoglycemia precludes maintenance of euglycemia over a lifetime of diabetes and thus full realization of the long-term vascular benefits of glycemic control.

\section{Functional brain failure}

Recent interest in alternative brain fuels (including lactate derived from glucose largely within the brain; refs. 4-6) notwithstanding, glucose is an obligate metabolic fuel for the brain under physiological conditions (7). Because the brain cannot synthesize glucose or store substantial amounts as glycogen in astrocytes, the brain requires a virtually continuous supply of glucose from the circulation. Facilitated diffusion of glucose from the blood into the brain is a direct function of the arterial plasma glucose concentration. The rate of blood-to-brain glucose transport exceeds the rate of brain glucose metabolism at normal (or elevated) plasma glucose levels, but it falls and becomes limiting to brain glucose metabolism when arterial glucose concentrations fall to low levels (8). Thus, hypoglycemia causes brain fuel deprivation and, as a result, functional brain failure.

The sequence of responses to falling plasma glucose concentrations (1) is illustrated in Figure 1. Initially, declining plasma glucose levels activate defenses against hypoglycemia. Physiological defenses normally include decrements in pancreatic $\beta$ cell insulin secretion as glucose levels decline within the physiological postabsorptive plasma glucose concentration range (approximately $3.9-6.1 \mathrm{mmol} / \mathrm{l}[70-110 \mathrm{mg} / \mathrm{dl}])$. The glycemic threshold for decreased insulin secretion is approximately $4.5 \mathrm{mmol} / \mathrm{l}(81$ $\mathrm{mg} / \mathrm{dl}$ ). Increments in pancreatic $\beta$ cell glucagon and adrenomedullary epinephrine secretion (among other neuroendocrine responses) normally occur as glucose levels fall just below the physiological range (threshold equal to approximately 3.8 $\mathrm{mmol} / \mathrm{l}[68 \mathrm{mg} / \mathrm{dl}])$. If these defenses fail to abort the hypoglycemic episode, lower glucose levels trigger a more intense sympathoadrenal response that causes neurogenic (or autonomic) symptoms; neuroglycopenic symptoms occur at about the same glucose level (threshold equal to approximately $3.0 \mathrm{mmol} / \mathrm{l}(54 \mathrm{mg} / \mathrm{dl})$. The perception of symptoms, particularly neurogenic symptoms, prompts the behavioral defense, the ingestion of food. If all of these defenses fail, lower glucose levels cause overt functional brain failure that 


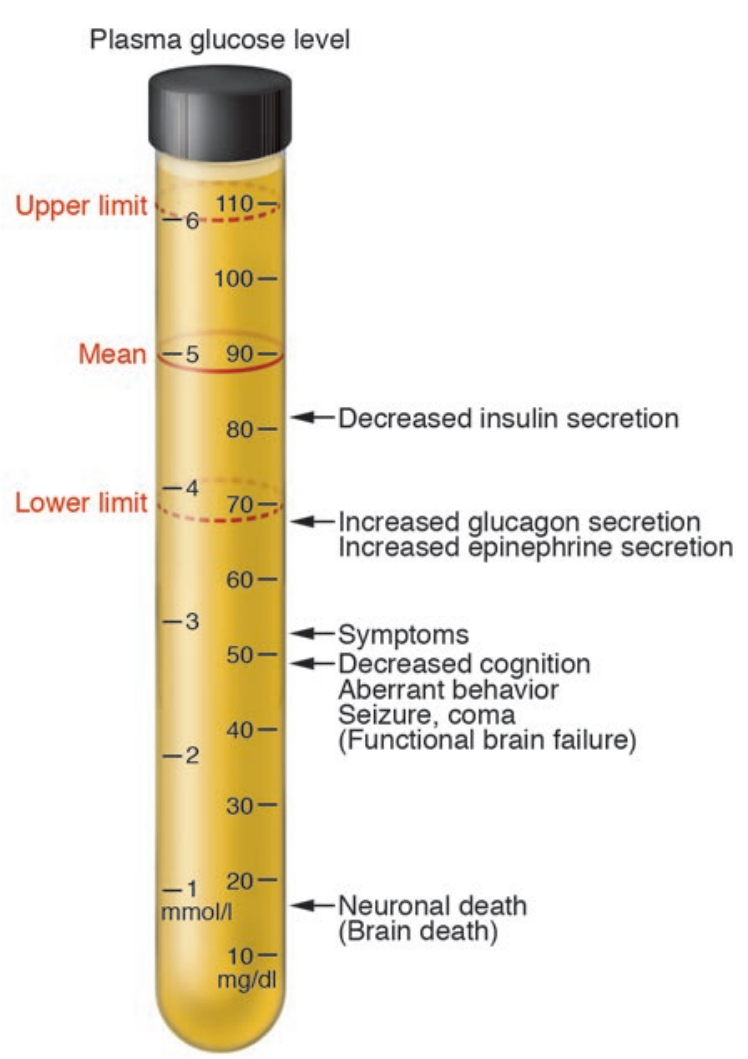

can progress from measurable cognitive impairments (threshold equal to approximately $2.8 \mathrm{mmol} / 1$ [50 mg/dl]) to aberrant behaviors, seizure, and coma. Coma can occur at glucose levels in the range of 2.3-2.7 mmol/1 (41-49 mg/dl) (9) as well as at lower glucose levels. All of these responses are typically corrected after the plasma glucose concentration is raised.

Episodes of hypoglycemia are a fact of life for most people with T1DM and many with advanced T2DM (3). In T1DM, plasma glucose concentrations may be less than $2.8 \mathrm{mmol} / 1(50 \mathrm{mg} / \mathrm{dl})$ as much as $10 \%$ of the time; the average patient suffers two episodes of symptomatic hypoglycemia per week and one episode of severe, temporarily disabling hypoglycemia per year. Although iatrogenic deaths do result from the adverse effects of drug therapy (9, 10) (the mechanisms are unclear but could include cardiac arrhythmias), seemingly complete recovery from hypoglycemiainduced functional brain failure after the plasma glucose concentration is raised is the rule $(3,11)$. Permanent neurological damage is rare (11).

\section{Brain death}

Profound, prolonged hypoglycemia can cause brain death. In studies of insulin- induced hypoglycemia in monkeys, 5-6 hours of blood glucose concentrations of less than $1.1 \mathrm{mmol} / \mathrm{l}(20 \mathrm{mg} / \mathrm{dl})$ were required for the regular production of neurological damage (12); the average blood glucose level was $0.7 \mathrm{mmol} / 1$ (13 mg/dl). Fortunately, hypoglycemia of that magnitude and duration occurs rarely in people with diabetes.

The mechanisms of the common, hypoglycemia-induced functional brain failure and of the rare, hypoglycemia-induced brain death that occurs at very low, and at least in primates prolonged, plasma glucose concentrations (Figure 1) differ. The former is the result of brain fuel deprivation per se, but the latter is not. As summarized by Suh and colleagues in their study reported in this issue of the JCI (13), a variety of mechanisms are thought to be involved in the pathogenesis of hypoglycemic neuronal death. These include glutamate release and activation of neuronal glutamate receptors, production of reactive oxygen species, neuronal zinc release, activation of poly(ADP-ribose) polymerase, and mitochondrial permeability transition.

In their current report, Suh and colleagues (13) describe additional studies of the mechanisms of hypoglycemia-induced neuronal necrosis. Based on systematic cell culture and in vivo rodent studies of glucose

\section{Figure 1}

Sequence of responses to falling arterial plasma glucose concentrations. The solid horizontal line indicates the mean and the dashed horizontal lines the upper and lower limits of physiological postabsorptive plasma glucose concentrations in humans. The glycemic thresholds for decrements in insulin secretion, increments in glucagon and epinephrine secretion, symptoms, and decrements in cognition have been defined in healthy humans (1) (see text). Those for seizure and coma and for neuronal death are extrapolated from clinical observations of humans (9) and studies in monkeys (12) as well as in other experimental animals (13-15). In this issue of the $\mathrm{JCl}$, Suh and colleagues (13) report that glucose reperfusion increased brain neuronal death in their rodent model of profound hypoglycemia.

deprivation followed by glucose provision, they provide evidence that hypoglycemic superoxide production and neuronal death are increased by NADPH oxidase activation during glucose reperfusion. These effects were reduced by an inhibitor of NADPH oxidase, deficiency of a subunit of the enzyme, and blockade of NADPH regeneration, among other findings. Notably, superoxide formation and neuronal death increased with increasing glucose concentrations during the posthypoglycemic reperfusion period. That finding is generally consistent with earlier findings by these investigators (14) and by others (15).

In order to reproducibly cause the study endpoints, including neuronal death, these studies (13) were generally performed at glucose concentration extremes. In the cell culture studies, glucose deprivation conditions were established by the use of a medium containing no glucose, while conditions of glucose provision were established by adding glucose to the medium at 10.0 $\mathrm{mmol} / 1$ (180 mg/dl), several-fold greater than normal brain extracellular fluid glucose concentrations. In the in vivo studies, blood glucose concentrations averaged 0.4 $\mathrm{mmol} / \mathrm{l}(7 \mathrm{mg} / \mathrm{dl})$, causing an isoelectric EEG, during hypoglycemia and approximately $7.5 \mathrm{mmol} / \mathrm{l}(135 \mathrm{mg} / \mathrm{dl})$ during glu- 
cose reperfusion that was documented to cause detrimental effects. Superoxide production, and presumably neuronal death, occurred as a result of hypoglycemia, but these occurred to a greater extent with glucose reperfusion, less so when posthypoglycemic blood glucose concentrations were raised to the range of $1.0-2.0 \mathrm{mmol} / 1$ $(18-36 \mathrm{mg} / \mathrm{dl})$ than when they were raised to the range of $5.0-10.0 \mathrm{mmol} / \mathrm{l}(90-180$ $\mathrm{mg} / \mathrm{dl}$ ). Studies involving less profound hypoglycemia were not reported.

The distinction between the common hypoglycemia-induced functional brain failure and the rare hypoglycemia-induced brain death drawn here is admittedly arbitrary. Plasma glucose concentrations of less than $1.0 \mathrm{mmol} / 1$ (18 mg/dl) occur occasionally in people with diabetes (9), and dying brain cells, presumably neurons, have been reported following episodes of hypoglycemia at plasma glucose levels of $1.7-1.9 \mathrm{mmol} / \mathrm{l}(30-35 \mathrm{mg} / \mathrm{dl})$ - but not following episodes of hypoglycemia at plasma glucose levels of $2.5 \mathrm{mmol} / 1$ $(45 \mathrm{mg} / \mathrm{dl})-$ in rats $(16)$. Thus, it could be reasoned that these categories are not binary and that there is a continuous spectrum with increasing risk of neuronal death at progressively lower plasma glucose concentrations. Nonetheless, seemingly complete recovery follows the vast majority of episodes of clinical hypoglycemia.

The appropriate clinical extrapolation of these data is not entirely clear. As the authors point out (13), plasma glucose concentrations must be raised in hypoglycemic patients. In the common clinical setting of hypoglycemia-induced functional brain failure, plasma glucose levels should be raised into the physiological range promptly with the expectation that recovery of brain function will follow. At this point there is no clear evidence that posttreatment hyperglycemia is detrimental to recovery, but there is no reason to think it is beneficial in that setting. On the other hand, undertreatment will delay recovery. In the rare clinical setting of profound, prolonged hypoglycemia, where the risk of neuronal death is higher, the data suggest that plasma glucose levels should be raised cautiously with avoidance of hyperglycemia (13-15). Nonetheless, it would seem reasonable to raise the plasma glucose level into the physiological range (e.g., >3.9 mmol/1 [70 mg/dl]) promptly. Clearly, additional studies of this important issue are needed.

\section{Acknowledgments}

The author's work cited was supported, in part, by US Public Health Service/NIH grants R37 DK27085, M01 RR00036, P60 DK20579, and T32 DK07120 and by a fellowship award from the American Diabetes Association. Janet Dedeke prepared this manuscript.

Address correspondence to: Philip E. Cryer, Campus Box 8127, Washington University School of Medicine, 660 South Euclid Avenue, St. Louis, Missouri 63110, USA. Phone: (314) 362-7635; Fax: (314) 3627989; E-mail: pcryer@wustl.edu.

1. Cryer, P.E. 2001. The prevention and correction of hypoglycemia. In Handbook of physiology. Section 7 The endocrine system. Volume 2, The endocrine pancreas and regulation of metabolism. L.S. Jefferson and A.D. Cherrington, editors. Oxford University Press. New York, New York, USA. 1057-1092.

2. Cryer, P.E. 2003. Glucose homeostasis and hypoglycemia. In Williams textbook of endocrinology. 10th edition. P.R. Larsen, H. Kronenberg, S. Melmed, and K. Polonsky, editors. Saunders/Elsevier. Philadelphia, Pennsylvania, USA. 1585-1618.

3. Cryer, P.E. 2004. Diverse causes of hypoglycemiaassociated autonomic failure in diabetes. N. Engl. J. Med. 350:2272-2279.

4. Aubert, A., Costalat, R., Magistretti, P.J., and Pellerin, L. 2005. Brain lactate kinetics: modeling evi- dence for neuronal lactate uptake upon activation. Proc. Natl. Acad. Sci. U. S. A. 102:16448-16453.

5. Schurr, A. 2006. Lactate: the ultimate cerebral oxidative energy substrate. J. Cerebr. Blood Flow Metab. 26:142-152.

6. Lubow, J.M., et al. 2006. Brain oxygen utilization is unchanged by hypoglycemia in normal humans: lactate, alanine, and leucine uptake are not sufficient to offset energy deficit. Am. J. Physiol. Endocrinol. Metab. 290:E149-E153.

7. Clarke, D.D., and Sokoloff, L. 1994. Circulation and energy metabolism of the brain. In Basic neurochemistry: molecular, cellular and medical aspects. 5 th edition. G. Siegel, B. Agranoff, R.W. Albers, and P. Molinoff, editors. Raven Press. New York, New York, USA. 645-680.

8. Blomqvist, G., et al. 1991. Facilitated transport of glucose from blood to brain in man and the effect of moderate hypoglycaemia on cerebral glucose utilization. Eur. J. Nucl. Med. 18:834-837.

9. Ben-Ami, H., Nagachandran, P., Mendelson, A., and Edoute, Y. 1999. Drug-induced hypoglycemic coma in 102 diabetic patients. Arch. Intern. Med. 159:281-284.

10. Laing, S.P., et al. 1999. The British Diabetic Association Cohort Study. II. Cause-specific mortality in insulin-treated diabetes mellitus. Diabet. Med. 16:466-471.

11. The Diabetes Control and Complications Trial Research Group. 1996. Effects of intensive therapy on neuropsychological function in adults in the Diabetes Control and Complications Trial. Ann. Int. Med. 124:379-388.

12. Kahn, K.J., and Myers, R.E. 1971. Insulin-induced hypoglycaemia in the non-human primate. I. Clinical consequences. In Brain bypoxia. J.B. Brierly and B.S. Meldrum, editors. William Heinemann Medical Books Ltd. London, United Kingdom. 185-194.

13. Suh, S.W., Gum, E.T., Hamby, A.M., Chan, P.H., and Swanson, R.A. 2007. Hypoglycemic neuronal death is triggered by glucose reperfusion and activation of neuronal NADPH oxidase. J. Clin. Invest. 117:910-918. doi:10.1172/JCI30077.

14. Suh, S.W., Gum, E.T., Hamby, A.M., Chan, P.H., and Swanson, R.A. 2003. Hypoglycemic neuronal death and cognitive impairment are prevented by poly(ADP-ribose)polymerase inhibitors administered after hypoglycemia. J. Neurosci. 23:10681-10690.

15. de Courten-Myers, G.M., et al. 2000. Hypoglycemic brain injury: potentiation from respiratory depression and injury aggravation from hyperglycemic treatment overshoots. J. Cereb. Blood Flow Metab. 20:82-92.

16. Tkacs, N.C., Pan, Y., Raghupathi, R., Dunn-Meynell, A.A., and Levin, B.E. 2005. Cortical Fluoro-Jade staining and blunted adrenomedullary response to hypoglycemia after noncoma hypoglycemia in rats. J. Cereb. Blood Flow Metab. 25:1645-1655. 